\title{
The Role of G Protein Alpha Subunits in the Infection Process of the Gray Mold Fungus Botrytis cinerea
}

\author{
Christian Schulze Gronover, Daniela Kasulke, Paul Tudzynski, and Bettina Tudzynski \\ Westfälische Wilhelms-Universität Münster, Institut für Botanik, Schloßgarten 3, D-48149 Münster, Germany \\ Submitted 18 April 2001; Accepted 6 July 2001.
}

\begin{abstract}
To identify signal transduction pathways of the gray mold fungus Botrytis cinerea involved in host infection, we used heterologous hybridization and a polymerase chain reaction (PCR)-based approach to isolate two genes (bcg1 and bcg2) encoding $\alpha$ subunits of heterotrimeric GTP-binding proteins. Both genes have homologues in other fungi: $b c g 1$ is a member of the $G \alpha_{i}$ class, whereas bcg 2 has similarities to the magC gene of Magnaporthe grisea and the gna-2 gene of Neurospora crassa. Reverse-transcription (RT)PCR experiments showed clearly that both genes are expressed at very early stages in infected bean leaves. Gene replacement experiments were performed for both genes. bcg1 null mutants differ in colony morphology from the wild-type strain, do not secrete extracellular proteases, and show clearly reduced pathogenicity on bean and tomato. Conidia germination and penetration of plant tissue is not disturbed in bcgl mutants, but the infection process stops after formation of primary lesions. In contrast, $b c g 2$ mutants show wild-type colony morphology in axenic culture and are only slightly reduced in pathogenicity. Complementation of bcgl mutants with the wild-type gene copy led to the full recovery of colony morphology, protease secretion, and pathogenicity on both host plants. Application of exogenous cyclic AMP restored the wild-type growth pattern of bcgl mutants, but not the protease secretion, implicating an essential role of BCG1 in different signaling pathways.
\end{abstract}

Botrytis cinerea Pers.:Fr., anamorph of Botryotinia fuckeliana (de Bary) Whetzel, is a widely occurring plant pathogen infecting fruits, flowers, or green tissues of more than 200 plant species. It does not need specific infection structures, such as appressoria, but seems to be able to directly penetrate healthy plant tissues. The mechanism of penetration and the process involved in the establishment of plant infection is not yet well understood. Cell wall-degrading enzymes (ten Have et al. 1998), phytotoxins (Rebordinos et al. 1996), active oxygen species (Liu et al. 1998; von Tiedemann 1997), or membrane transporters for secretion of plant defense compounds (Schoonbeek et al. 2001) have been implicated as pathogenicity factors for $B$. cinerea. A basic requirement for pathogenicity is the ability to sense and respond to extracellular signals.

Corresponding author: Bettina Tudzynski; Telephone: (0049)251.83-24801; Fax: (0049)251.83-23823; E-mail: Bettina.Tudzynski@uni-muenster.de
Therefore, the analysis of signal chains that control whole sets of genes involved in the colonization of plant tissue could give an understanding of the complex interaction mechanisms. Heterotrimeric GTP-binding proteins are a family of regulatory proteins that play an important role in the transduction of signals from the environment to target genes in eukaryotic cells. Since $G \alpha$ proteins are the first components of signaling chains, receiving the signals directly from receptors, they might be involved in regulating plant infection processes. In the past years, it has been shown that $G$ protein-mediated signal transduction is involved in many aspects of host-fungal pathogen interactions on both the fungal and the plant sides. Choi and colleagues (1995) reported that, in the chestnut blight fungus Cryphonectria parasitica, one of two genes encoding $\mathrm{G}$ protein $\alpha$ subunits, cpgl, is required for both virulence and female fertility, whereas the second gene, $c p g 2$, has no obvious role in pathogenicity (Gao and Nuss 1996). In the rice pathogen Magnaporthe grisea, three $\mathrm{G} \alpha$ protein-encoding genes ( $m a g A, m a g B$, and $m a g C$ ) were cloned. One of them, $m a g B$, is required for both appressorium formation on rice leaves and perithecium production, whereas mutations in the magA and $m a g C$ genes did not affect the virulence of the fungus (Liu and Dean 1997). A G $\alpha$ subunit-encoding gene of the corn pathogen Cochliobolus heterostrophus, cgal, also belonging to the $\mathrm{G \alpha}_{\mathrm{i}}$ class, is required for appressorium formation and mating (Horwitz et al. 1999). Of four $\mathrm{G} \alpha$ protein-encoding genes (gpal to gpa4) cloned from the corn pathogen Ustilago maydis, only the mutation of gpa3 caused mating deficiency as well as inability to infect the host plant (Regenfelder et al. 1997). Interestingly, gpa3 seems to participate in different signaling pathways with and without the involvement of pheromones.

In the pathogenic fungus Colletotrichum trifolii, the causal agent of alfalfa anthracnose, a $\mathrm{G} \alpha$ subunit-encoding gene, ctg1, is involved in conidial germination and appressorial formation on both glass slides and alfalfa leaves, as well as in the infection of the host plant (Truesdell et al. 2000).

In this study, we have cloned and characterized two G $\alpha$ subunit-encoding genes from the gray mold fungus $B$. cinerea. In contrast to all the other plant pathogens described above, $B$. cinerea is a broad-host-range necrotrophic pathogen that attacks more than 200 plant species, and the mechanisms to achieve this are not yet understood. Therefore, cloning and deletion of the corresponding genes opens new perspectives for the identification of pathogenicity genes of the gray mold B. cinerea. 

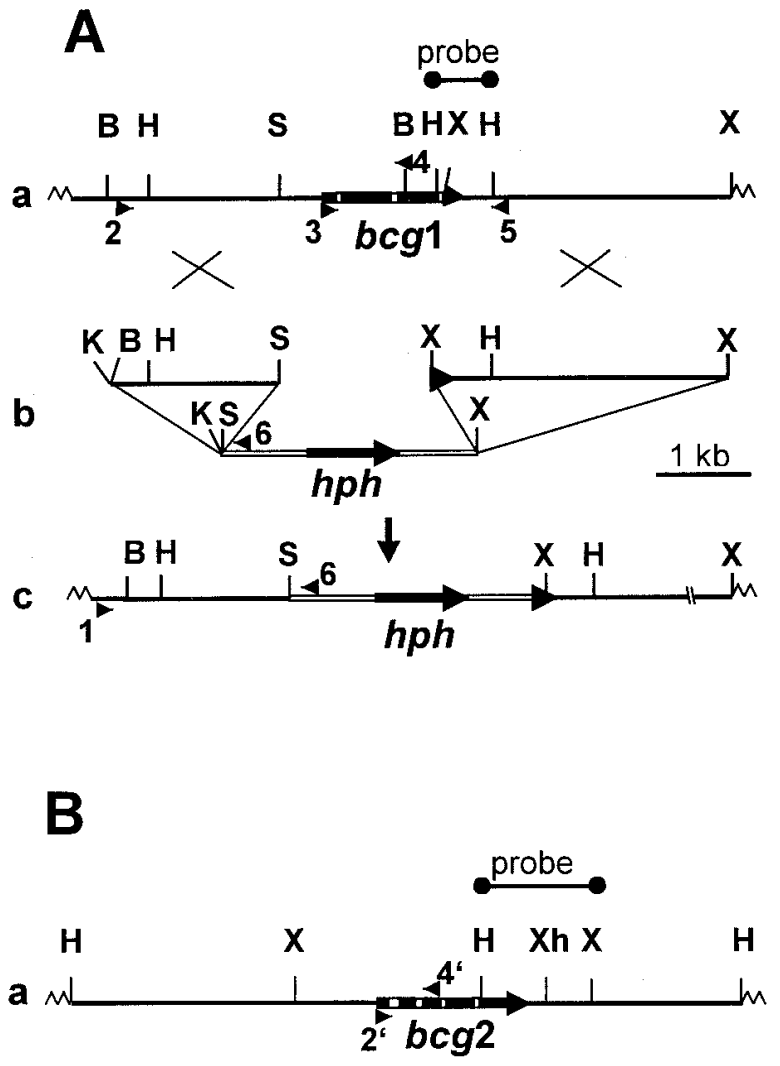

b
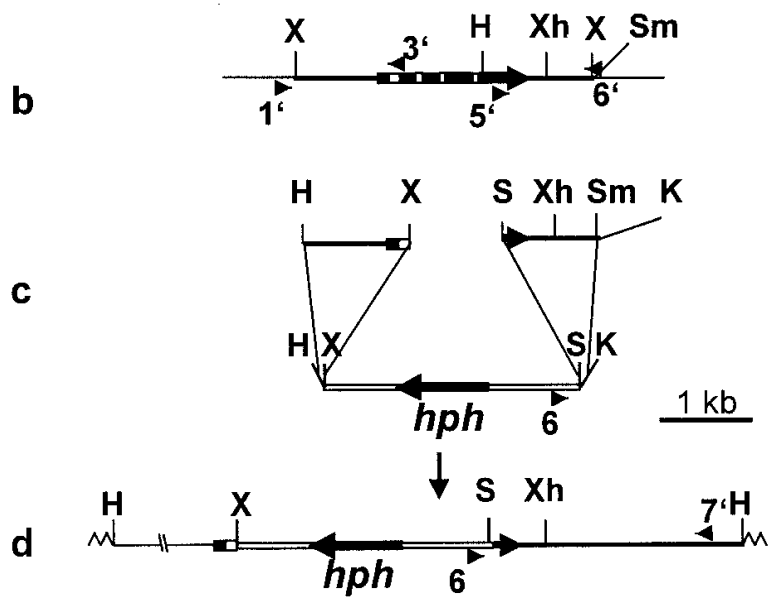

Fig. 1. Restriction maps and gene replacement strategy for A, $b c g 1$ and $\mathbf{B}, b c g 2$. A: a, Physical maps of the genomic locus of $b c g 1 ; \mathbf{b}$, cloning of the KpnI/SalI and $\mathrm{XbaI}$ fragments into the corresponding sites of the vector pLOF1A; and c, genomic region after the homologous integration of the transformation fragment into the bcgl locus by double cross-over. B: a, Physical map of the genomic locus of bcg2; $\mathbf{b}$, amplification of the left and right flanks by polymerase chain reaction (PCR), the position of primers $1^{\prime}, 3^{\prime}, 5^{\prime}$, and $6^{\prime}$ are shown; c, cloning of the PCR fragments that were cut from the PCR cloning vector PCR2.1 with HindIII/XbaI and SalI/KpnI into the vector pLOF1A; and d, genomic region after the homologous integration of the transformation fragment into the $b c g 2$ locus. Accession numbers in the EMBL database: $b c g 1, \mathrm{Y} 18436$, and $b c g 2$, Y18437. Arrows indicate position of primers used for reverse transcription-PCR, construction of replacement vectors, and analysis of transformants (details in Material and Methods). $\mathrm{K}=K p n \mathrm{I}, \mathrm{B}=\operatorname{Bam} \mathrm{HI}, \mathrm{H}=$ HindIII, S = SalI, X =XbaI, Xh =XhoI, and Sm $=S m a \mathrm{I}$.

\section{RESULTS}

Cloning of bcg1 and bcg2.

The $b c g 1$ gene was isolated from a genomic $\lambda$-EMBL3 library of strain SAS56 (Quidde et al. 1999) with the cpg-1 gene from $C$. parasitica as a heterologous probe. Two of the thirty-six strongly hybridizing plaques that were identified in a first round $(\lambda 3 / 1$ and $\lambda 4 / 1)$ were purified in a second screening round. A 2.4-kb SalI fragment and a 3.0-kb BamHI fragment (both of which hybridized with the probe) were cloned into pUC19 from $\lambda 3 / 1$ and $\lambda 4 / 1$, respectively, and sequenced.

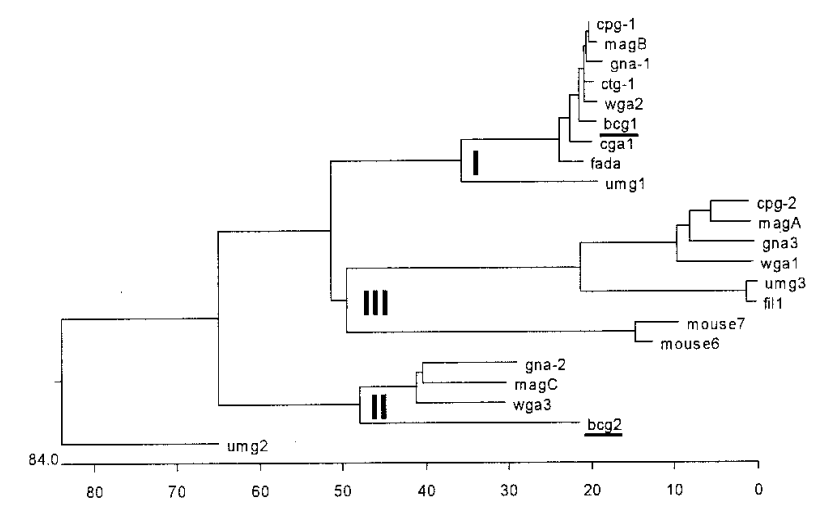

Fig. 2. The phylogenetic relationship between Botrytis cinerea bcgl and $b c g 2$ and other $\mathrm{G} \alpha$ proteins. The cladogram was generated based on multiple sequence alignment (program MEGALIN; DNASTAR Inc., Madison, WI, U.S.A.) of the following $\mathrm{G} \alpha$ proteins: Cryphonectria parasitica (cpg-1 and cpg-2) (Choi et al. 1995); Neurospora crassa (gna1 and gna2) (Turner and Borkovich 1993); N. crassa (gna3) (Kays et al. 2000); Ustilago maydis (umg1 and umg3) (Regenfelder et al. 1997); Magnaporthe grisea (magA, magB, and magC) (Liu and Dean 1997); U. hordei (fil1) (Lichter and Mills 1997); Aspergillus nidulans (fadA) (Yu et al. 1996); Rosellinia necatrix (RGA-1, RGA-2, and RGA3) (Aimi et al. 2001); Mus musculus (mouse 6 and 7) (Strathmann et al. 1989); Cochliobolus heterostrophus (cga1) (Horwitz et al. 1999), and Colletotrichum trifolii (ctg-1) (Truesdell et al. 2000).

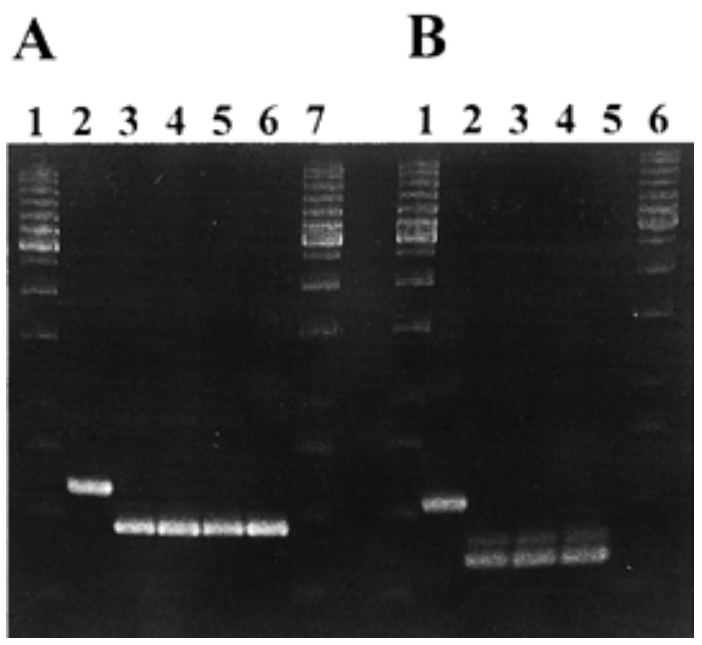

Fig. 3. Reverse-transcription-polymerase chain reaction analysis of $\mathbf{A}$, bcg1 and $\mathbf{B}, b c g 2$ expression in plants. Bean leaves were inoculated with conidia of the wild-type strain B05.10, and RNA was prepared from infected tissue after $12,24,36$, and 48 h postinoculation. A, Lanes 1 and 7 and $\mathbf{B}, 1$ and 6,1-kb ladder; 2, genomic DNA was used as template; 3, 12 hpi; 4, 24 hpi; 5, 36 hpi; and A, 6, 48 hpi. 
The SalI clone, pbcg-1, showed a predicted open reading frame of 1,261 bp (353 amino acids) interrupted by three putative introns. The intron positions were confirmed by comparison of cDNA and genomic DNA clones. Figure 1A shows the restriction map of the $B$. cinerea genomic DNA that hybridized to the cpgl probe. The deduced amino acid sequence showed $96 \%$ identity with CPG-1 from C. parasitica and $95 \%$ with MAGB from $M$. grisea.

For the cloning of additional G $\alpha$-encoding genes, degenerated primers (Ga-1 and Ga-2) were used for polymerase chain reaction (PCR) amplification of $B$. cinerea genomic DNA and cDNA. A band of the expected size (180 bp) was amplified and cloned into the PCR2.1 vector for sequence analysis. Two different sequences were identified, one of which was the already-cloned bcgl gene and the other a second G $\alpha$-encoding gene, $b c g 2$. The $B$. cinerea EMBL3 library was screened with the $b c g 2$ fragment as a probe, and eight hybridizing phages were isolated and purified. A 4.3-kb HindIII fragment and a 3.0-kb XbaI fragment were cloned into pUC19, sequenced on both strands, and compared with the corresponding cDNA clone. Figure $1 \mathrm{~B}$ shows the restriction map of gene $b c g 2$. An open reading frame of $1,367 \mathrm{bp}$ encodes a protein of 354 amino acids with $46 \%$ identity to BCG1. The coding sequence includes four introns, whose positions were determined by comparison of cDNA and genomic DNA clones and by the presence of consensus splicing sites.

A phylogenetic tree of fungal $G \alpha$ subunits, constructed on the basis of amino acid sequences, revealed that BCG1 groups into the class of $\mathrm{G \alpha}_{\mathrm{i}}$ subunits (I) together with cpgl from C. parasitica, magB from $M$. grisea, ctg- 1 from Colletotrichum trifolii, cgal from Cochliobolus heterostrophus, and gpa-1 from U. maydis. On the other hand, BCG-2 is a member of group II of $\mathrm{G} \alpha$ subunits, together with $\operatorname{magC}$ and gpa-2 (Fig. 2).

\section{Expression studies.}

In order to examine $b c g 1$ and $b c g 2$ expression during infection on bean leaves, reverse-transcription (RT)-PCR experiments were performed with RNA from necrotic leaf spots 12 , 24,36 , and $48 \mathrm{~h}$ postinoculation (hpi) using the primers 3 and 4 for $b c g 1$ and primers $2^{\prime}$ and $4^{\prime}$ for $b c g 2$ spanning the introncontaining regions of both genes (Fig. 1A and B). As shown in Figure 3, RT-PCR products of the expected sizes (464 and $344 \mathrm{bp}$, respectively) were obtained, clearly distinct from the DNAderived control PCRs. The products were proofed by sequencing. Both genes were already expressed at the beginning of symptom development (12 hpi) and were still active after $36 \mathrm{~h}$, bcgl even after $48 \mathrm{~h}$.
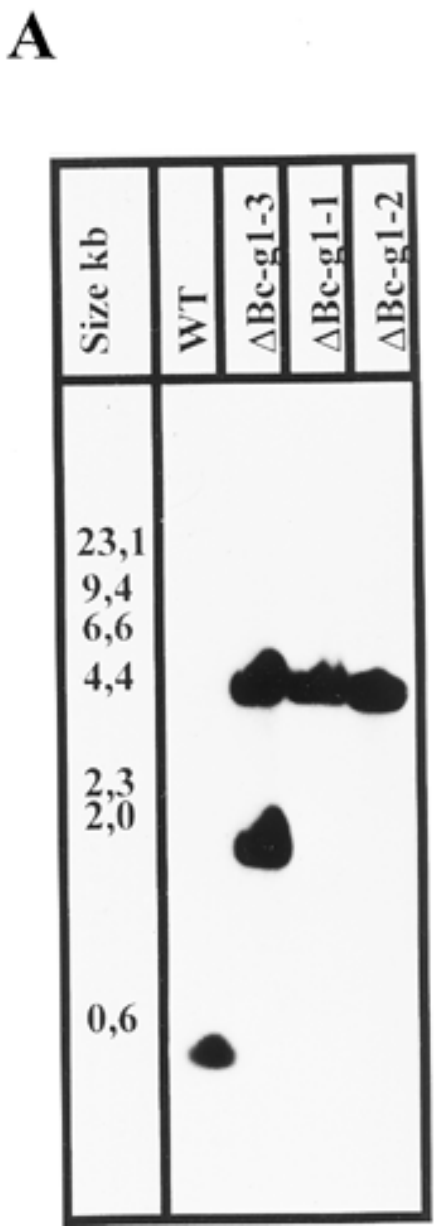

B

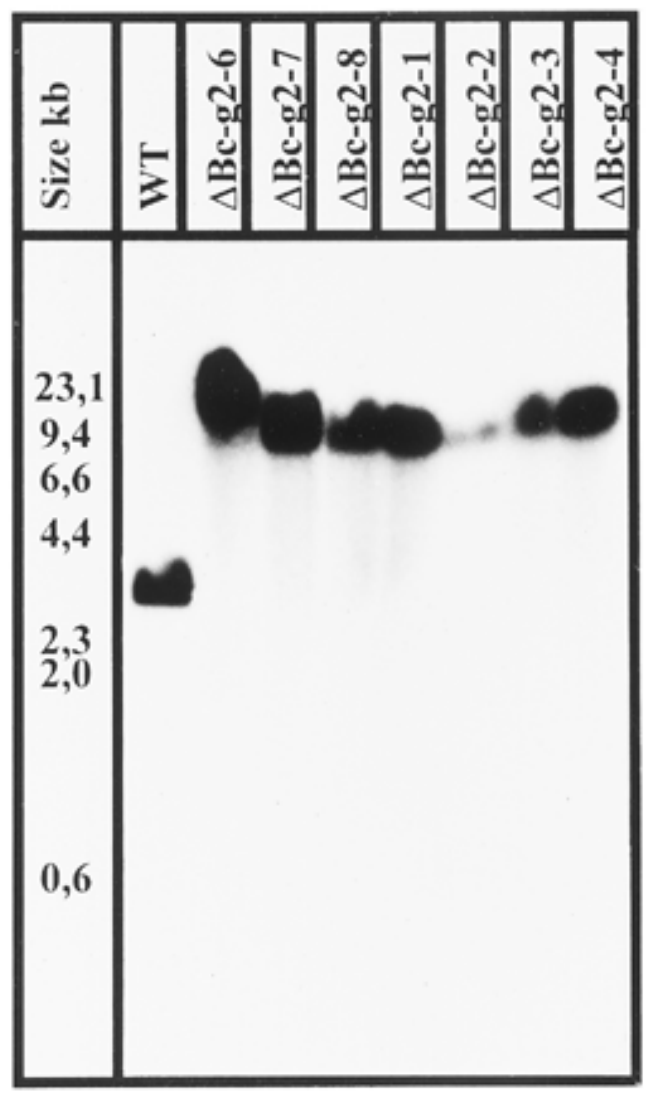

Fig. 4. Southern blot analysis of the wild-type and transformant strains. A, DNA of strain B05.10 and the transformants $\Delta \mathrm{bcg} 1-1, \Delta \mathrm{bcg} 1-2$, and $\Delta \mathrm{bcg} 1-3$ was restricted with HindIII and probed to the 575-bp bcg1-HindIII fragment. B, DNA of the wild-type strain B05.10 and the transformants $\Delta$ bcg2-6, $\Delta \mathrm{bcg} 2-7, \Delta \mathrm{bcg} 2-8, \Delta \mathrm{bcg} 2-1, \Delta \mathrm{bcg} 2-2, \Delta \mathrm{bcg} 2-3$, and $\Delta \mathrm{bcg} 2-4$ was restricted with HindIII and probed to the 810-bp HindIII/XhoI fragment of $b c g 2$. 
Targeted disruption of the B. cinerea bcg1 and bcg 2 genes.

For functional analysis of the $\mathrm{G} \alpha$ subunits, knock-out mutants were generated for both genes. Gene replacement vectors for $b c g 1(\mathrm{p} \Delta \mathrm{bcg} 1)$ and $b c g 2(\mathrm{p} \Delta \mathrm{bcg} 2)$ were constructed as illustrated in Figure 1. For $\mathrm{p} \Delta \mathrm{bcg} 1$, a 1.8-kb KpnI/SalI fragment of bcgl was cloned directly upstream of the oliC promoter (left flank); sequence analysis of this region did not reveal any open reading frame. A 3.0-kb XbaI fragment (right flank) was cloned adjacent to the $\operatorname{trpC}$ terminator of the vector $\mathrm{pLOF} 1 \mathrm{~A}$, containing the Escherichia coli hygromycin resistance gene.

A bcg2 replacement vector was constructed by cloning a $1.1-\mathrm{kb}$ PCR fragment of the $3^{\prime}$ region of $b c g 2$ into the $K p n \mathrm{I} / \mathrm{SalI}$ sites and a $1.6-\mathrm{kb} \mathrm{PCR}$ fragment of the $5^{\prime}$ region into the XbaI/HindIII sites of pLOF1A. Transformations were performed with the KpnI-linearized vector $\mathrm{p} \Delta \mathrm{bcg} 1$ and the SmaI/HindIII-digested replacement cassette of $\mathrm{p} \Delta \mathrm{bcg} 2$.

The hygromycin-resistant transformants were analyzed by PCR for homologous integration with primer 6 , binding in the resistance cassette, and $b c g 1$ - and $b c g 2$-specific primers 1 and $7^{\prime}$, respectively (Fig. 1B). Putative knock-out mutants yielding the expected diagnostic PCR fragments (data not shown) were

\section{A}

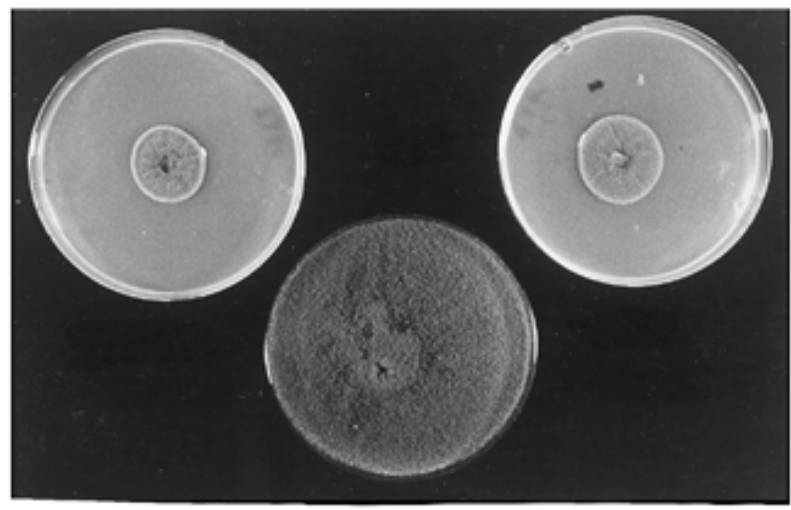

B

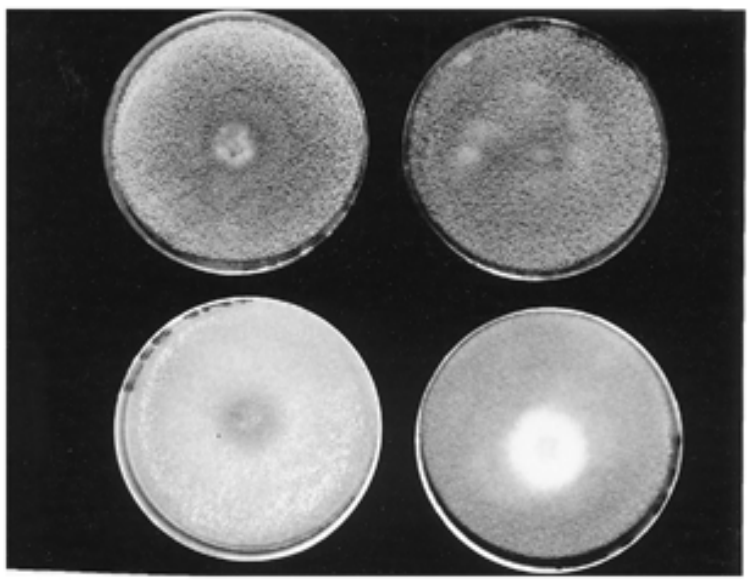

Fig. 5. Colony morphology of the wild-type strain B05.10 and the bcg 1 knock-out transformants on potato sucrose agar (PSA) A, without and $\mathbf{B}$, with $2 \mathrm{mM}$ cyclic AMP (cAMP). A, Strains $\Delta$ bcg1-1 and $\Delta$ bcg1-2 (upper row) and B05.10 (lower row) on PSA with $0.5 \%$ sucrose after 10 days of incubation. B, B05.10 (upper row) and $\Delta$ bcg1-1 (lower row) on PSA $(0.5 \%$ sucrose) without (left side) and with (right side) cAMP. purified several times by single-spore isolation. The gene replacement event was confirmed by Southern blot analysis. As shown in Figure 4A, in all three $b g c l$ transformants, the wildtype fragment $(0.5 \mathrm{~kb})$ is replaced, as expected, by a $4.3-\mathrm{kb}$ fragment, resulting from a double cross-over-mediated replacement event. Mutant $\Delta$ bcg1-3 contains an additional fragment, indicating an additional ectopic vector integration. All
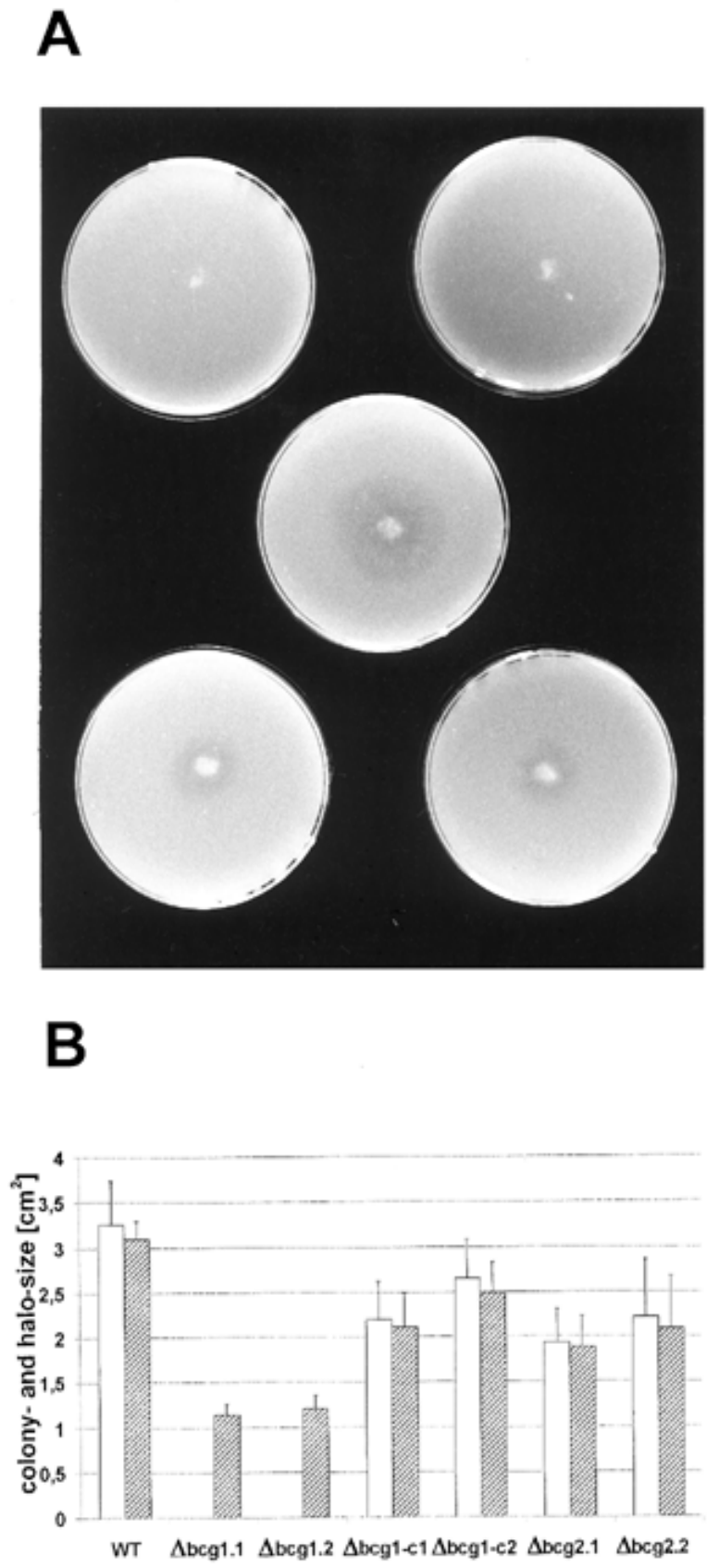

Fig. 6. Secretion of extracellular proteases on milk agar after 4 days of incubation. A, Upper row, $\Delta$ bcg1 transformants $\Delta$ bcg1-1 (left) and $\Delta$ bcg1-2 (right) (no secretion); middle row, wild-type strain B05.10; and lower row, $\Delta \mathrm{bcg} 2$ transformants $\Delta \mathrm{bcg} 2-1$ (left) and $\Delta \mathrm{bcg} 2-2$ (right). B, Colony size (white bar) and size of the transparent halo (hatched bar). The average and standard error for each value represent data from six independent experiments. 


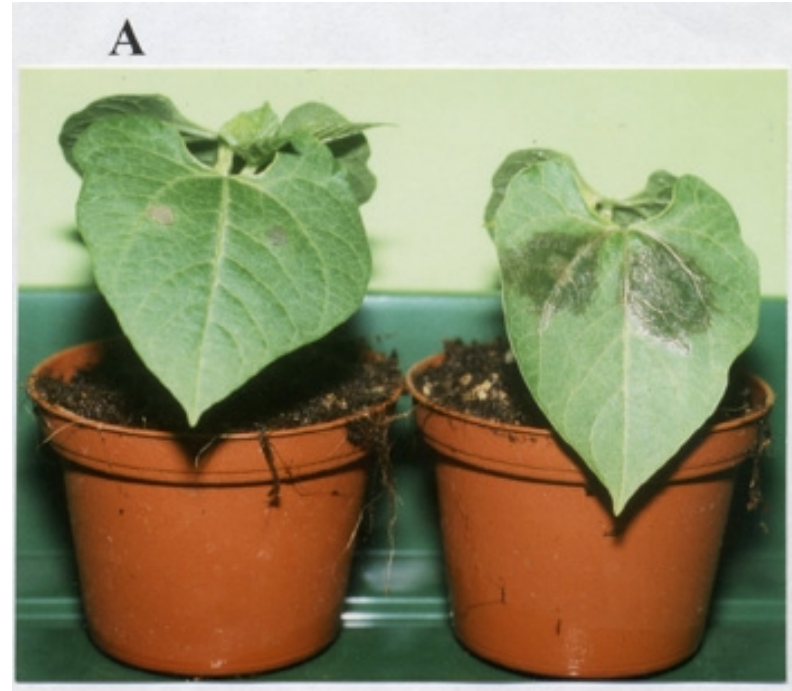

C

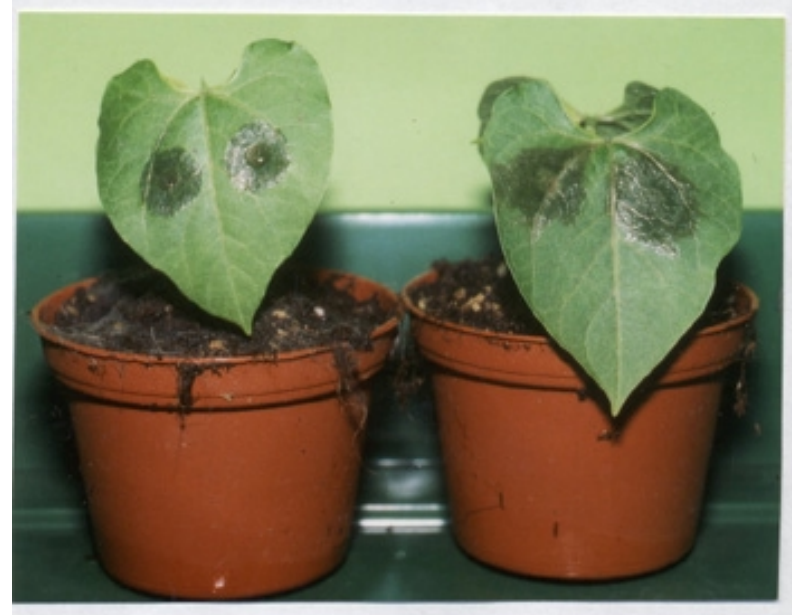

E

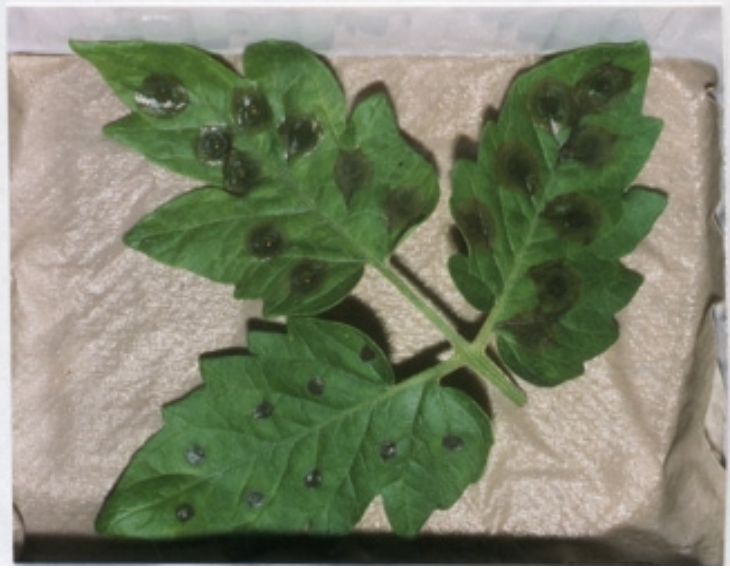

B

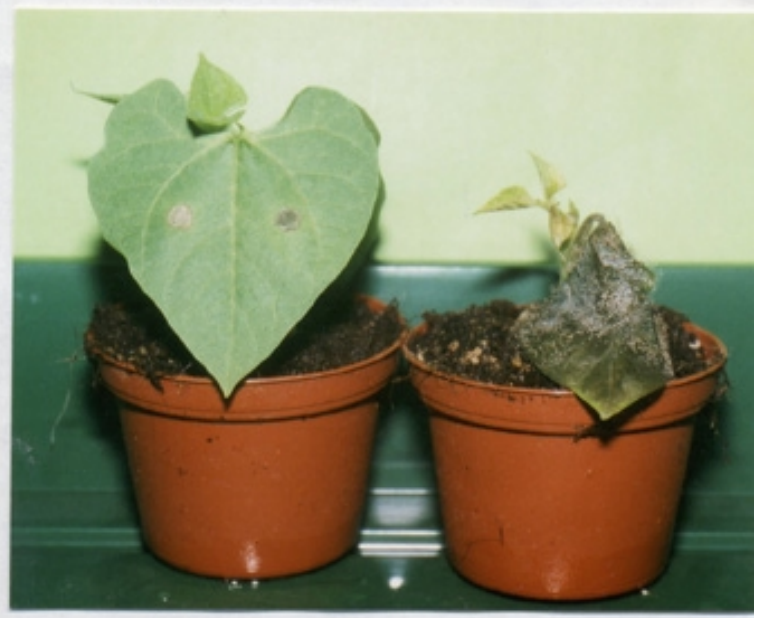

D

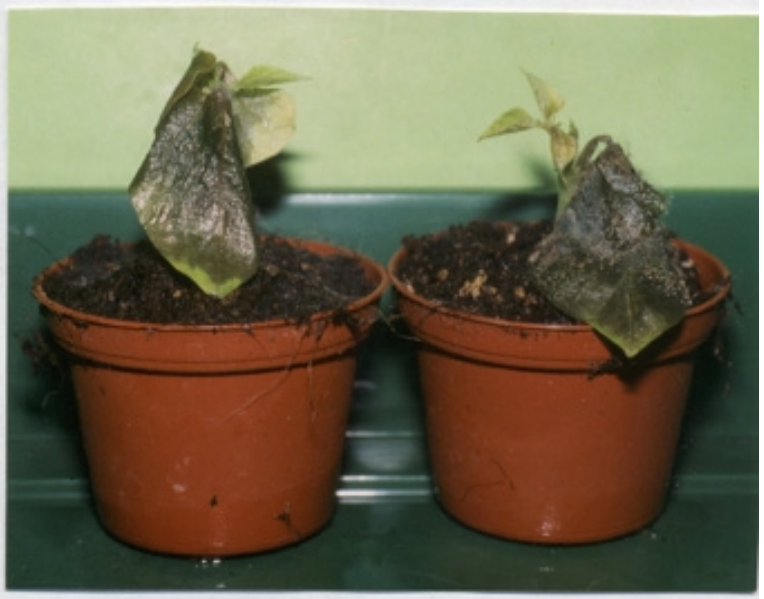

F

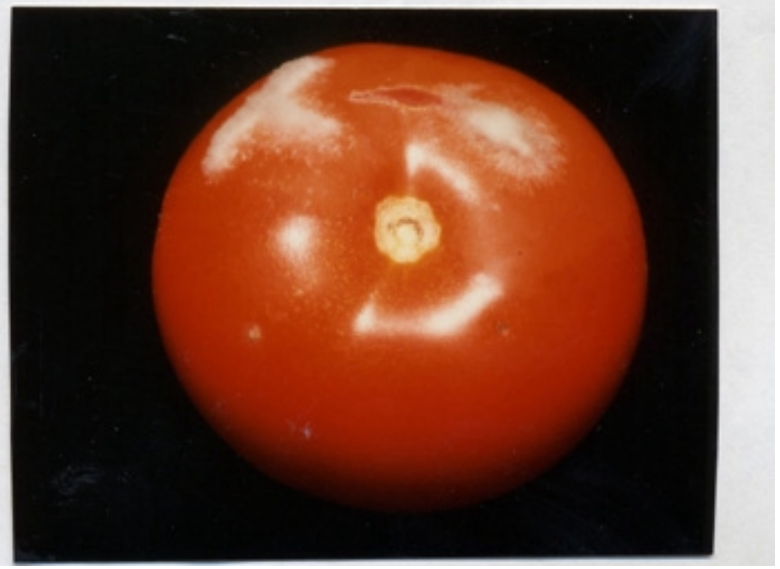

Fig. 7. Pathogenicity assays of Botrytis cinerea strains. A to D, Primary leaves of bean plants; $\mathbf{E}$, detached tomato leaves; and $\mathbf{F}$, tomato fruit were inoculated with conidia $\left(10^{5}\right)$ of $\mathbf{A}, \Delta \mathrm{bcg} 1-1$ (left plant) and the wild-type B05.10 (right plant) $48 \mathrm{~h}$ postinoculation (hpi); B, $\Delta \mathrm{bcg} 1-1$ (left plant) and B05.10 (right plant) 7 days postinoculation (dpi); C, $\Delta$ bcg2-1 (left plant) and B05.10 (right plant) 48 hpi; D, $\Delta$ bcg2-1 (left plant) and B05.10 (right plant) $7 \mathrm{dpi}$; E, $\Delta$ bcg1-1 (left leaf), B05.10 (middle leaf), and $\Delta$ bcg2-1 (right leaf) $7 \mathrm{dpi}$; and F, $\Delta$ bcg1-1 (bottom left), $\Delta$ bcg1-c1 (top left), and $\Delta$ bcg2-1 (top right) 7 dpi. 
bcg2 transformants tested (Fig. 4B) show the expected band shift, with no hints of additional ectopic integration. For further analyses, mutants $\Delta$ bcg1-1/2 and $\Delta$ bcg2-1/2 were chosen, which represent homokaryotic single-copy replacement transformants.

\section{Colony morphology}

and cyclic AMP (cAMP) feeding.

Colonies of $\Delta \mathrm{bcg} 1$ mutants, in contrast to $\Delta \mathrm{bcg} 2 \mathrm{mu}$ tants, show altered colony morphology in axenic culture compared with the recipient strain B05.10. When cultured on potato sucrose agar (PSA) $(0.5 \%$ sucrose), they grow slowly and have even colony margins. They show normal conidiation with an intense brownish grey pigmentation (Fig. 5A). After 10 days of incubation, colonies continue to grow with thin, nonsporulating mycelium (Fig. 5B, lower lane left).

To assess the effects of exogenously applied cAMP on colony morphology, the $\Delta \mathrm{bcg} 1$ mutants and the wild type were grown on PSA $(0.5 \%$ sucrose) supplemented with 2,5 , or $10 \mathrm{mM}$ cAMP. After a short phase of growth with white, nonsporulating mycelium, the wild-type colony morphology, including sporulation, was fully restored at cAMP concentrations of $2 \mathrm{mM}$, clearly demonstrating that $b c g l$ is involved in the cAMP signaling pathway (Fig. 5B).

\section{Extracellular protease assay.}

The wild-type strain B05.10 and the $\Delta \mathrm{bcg} 1$ and $\Delta \mathrm{bcg} 2 \mathrm{mu}-$ tants were analyzed for their ability to secrete extracellular proteases by incubation on casein agar. The strain B05.10 and the mutants $\Delta$ bcg2-1 and $\Delta$ bcg2-2 secreted proteases, resulting in the formation of transparent halos around the colonies. However, $\Delta$ bcg1-1 and $\Delta$ bcg1-2 have lost their ability to secrete extracellular proteases (Fig. 6A). Colony and halo size of all strains after 4 days of growth on milk agar is shown in Figure 6B. Corresponding tests for other extracellular enzyme activities (phenoloxidases, lipases, and pectinolytic enzymes) yielded no differences.

In order to show if the protease secretion can be restored by cAMP, bcg1-1 and bcg1-2 were incubated on milk agar complemented with $2 \mathrm{mM}$ cAMP. However, in contrast to colony morphology, protease secretion was not recovered by the addition of cAMP.
Infection assays on plants and tomato fruits.

Conidia of the strain B05.10 and the mutants $\Delta$ bcg1-1 and $\Delta$ bcg1-2, as well as $\Delta$ bcg2-1 and $\Delta$ bcg2-2, were dropped onto primary leaves of young bean plants. Primary necrotic lesions became visible for all three genotypes $24 \mathrm{hpi}$. After $48 \mathrm{~h}$, these lesions had enlarged on leaves infected with conidia of B05.10 and both $\Delta \mathrm{bcg} 2$ mutants; after 72 hpi on these leaves, spreading lesions were formed. In the infections with wild-type spores, these "secondary" lesions enlarged rapidly; the lesions caused by spores from $\Delta$ bcg2 mutants spread with slightly reduced speed. In contrast, the growth of primary necrotic lesions observed on leaves infected with conidia of $\Delta$ bcg1-1 and $\Delta$ bcg1-2 were completely arrested at $48 \mathrm{hpi}$, and spreading lesions were never observed. The infection assays were repeated four times, giving the same result (Fig. 7A to D). The same infection development was observed on detached tomato leaves: primary lesions became visible for all phenotypes 24 hpi and grew to spreading "secondary" lesions with spores from the wild type and the $\Delta \mathrm{bcg} 2$ mutants. For $\Delta$ bcgl-infected leaves, spreading lesions were never formed (Fig. 7E).

A pathogenicity assay was also performed by dropping conidia of the wild-type strain and $\Delta \mathrm{bcg} 1$ and $\Delta \mathrm{bcg} 2$ mutants onto the wounded (with a needle) surface of tomato fruits. In the case of strain B05.10 (data not shown) and $\Delta \mathrm{bcg} 2$ mutants, spreading lesions were observed, whereas only weak symptoms were observed on fruits infected with $\Delta$ bcg1 conidia (Fig. 7F).

These observations demonstrate that $b c g 1$, in contrast to $b c g 2$, might play a major role in the invasion of plant tissue after penetration.

\section{Morphology of the infection structures.}

Since spreading "secondary" lesions were never observed for knock-out mutants of $b c g l$, we wanted to know if the mutant hyphae are affected in their ability to penetrate healthy plant tissue or if they are able to penetrate but are then affected in the further invasion of plant tissue. Lesions from four different bean leaves were analyzed by scanning electron microscopy (SEM). As is seen in Figure 8, the mutants $\Delta$ bcg1-1 and $\Delta$ bcg1-2 were able to penetrate the cuticula.
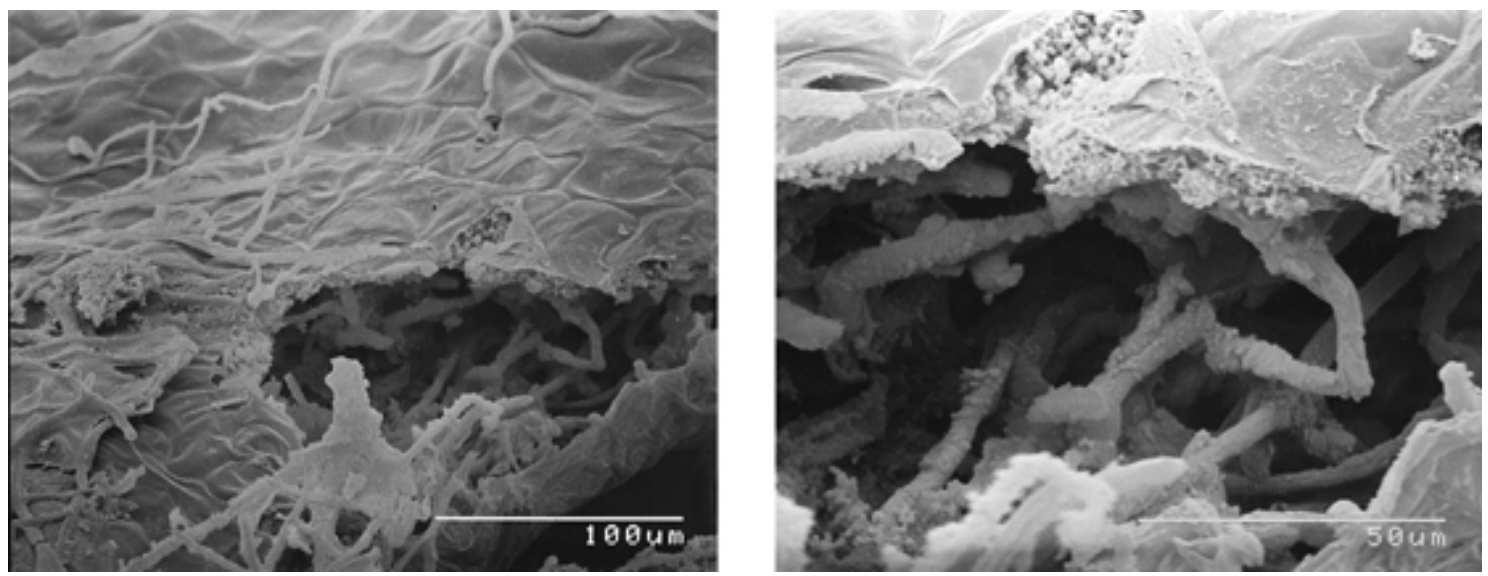

Fig. 8. Scanning electron microscopy of bean leaves infected with conidia of the strain $\Delta \mathrm{bcg} 1-172 \mathrm{~h}$ postinoculation. 


\section{Complementation analysis.}

Though the fact that several independent knock-out mutants of both genes show the same phenotype leaves no doubt that this phenotype is caused by the gene inactivation, we decided to confirm the severe phenotype linked to the inactivation of bcgl by a complementation approach. Mutant $\Delta$ bcg1-1 was transformed with the vector pbcg1+, containing a complete copy of bcgl (including a sufficient part of the $5^{\prime}$ noncoding region), and putative complementation transformants (selected for phleomycin resistance via the cotransformation vector pLOF2) were checked by PCR for the presence of a complete copy of bcgl (data not shown). Two of the transformants ( $\Delta \mathrm{bcg} 1-\mathrm{c} 1$ and $\Delta \mathrm{bcg} 1-\mathrm{c} 2$ ) showed full restoration of the wildtype phenotype with respect to vegetative growth, protease secretion, and pathogenicity on bean plants (data not shown). In Figure 7F, restoration of pathogenicity of $\Delta \mathrm{bcg} 1-\mathrm{c} 1$ on tomato fruits is demonstrated. Mutant $\Delta \mathrm{bcg} 1-\mathrm{c} 1$ was able to invade the tomato fruit with the same speed as the wild type (data not shown).

\section{DISCUSSION}

Here we describe the cloning, characterization, and targeted disruption of two $\mathrm{G} \alpha$ subunit-encoding genes of the gray mold B. cinerea.

Heterotrimeric $\mathrm{G}$ proteins are important for the regulation of diverse biological processes in filamentous fungi, such as sexual reproduction (Liu and Dean 1997; Regenfelder et al. 1997; Turner and Bokovich 1993), asexual development (Lichter and Mills 1997; Yu et al. 1996); and pathogenicity (Choi et al. 1995; Horwitz et al. 1999; Liu and Dean 1997; Regenfelder et al. 1997; Truesdell et al. 2000).

In mammalian systems, $\mathrm{G} \alpha$ subunits have been placed into different subfamilies based on their function and amino acid sequence similarity. Members of $\mathrm{G} \alpha_{\mathrm{s}}$ subgroup stimulate and members of $G \alpha_{i}$ subfamily inhibit adenylate cyclase, and $G \alpha_{o}$ subunits are involved in the regulation of potassium channels, phospholipase C, and cyclic GMP phosphodiesterase. A multiple alignment of the fungal $\mathrm{G} \alpha$ subunits revealed three major subgroups (I to III). Members of subgroups I and III can be related to the mammalian $\mathrm{G} \alpha_{\mathrm{i}}$ and $\mathrm{G} \alpha_{\mathrm{s}}$ proteins, respectively (Bölker 1998). Group II of fungal $\mathrm{G} \alpha$ proteins does not precisely correspond with any of the $\mathrm{G} \alpha$ subfamilies established for higher organisms.

Based on sequence similarities, BCG1 was grouped into fungal group I $\left(\mathrm{G \alpha}_{\mathrm{i}}\right)$, which is characterized by an N-terminal myristoylation site (MGXXXS); a $\mathrm{C}$ terminus with a pertussis toxin-catalyzed ADP-ribosylation site (CAAX), where $\mathrm{C}$ might be prenylated (Pennington 1994); and a P loop, one of the ATP/GTP-binding motifs with alanine in the second position. All members of this $\mathrm{G} \alpha_{i}$ group contain three introns in conserved positions of the open reading frame.

Together with MAGC in $M$. grisea, GPA2 from $U$. maydis, and GNA2 from Neurospora crassa, BCG2 belongs to subfamily II of fungal $\mathrm{G} \alpha$ proteins. The amino acid sequence at the $\mathrm{C}$ terminus, as well as the number and positions of introns, differ from those of subgroup I. Deletion mutants of the $\mathrm{magC}$ gene showed reduced conidia formation and interfered with ascospore development (Liu and Dean 1997). For the other members of this group, the function is still unknown.
For functional analysis of BCG1 and BCG2, both genes were inactivated by gene replacement. In the case of $b c g l$, about $500 \mathrm{bp}$ of the $5^{\prime}$ noncoding region were deleted together with the coding region. Since the entire 1.8 -kb upstream region used for the construction of the vector $\mathrm{p} \Delta \mathrm{bcg} 1$ does not contain an open reading frame, it is very unlikely that a neighborhood gene is affected by this deletion. As shown for the other fungal $\mathrm{G} \alpha_{i}$ proteins, BCG1 controls multiple functions, including vegetative growth, pigmentation, proteolytic activity, and pathogenicity. The changes in colony morphology are similar to those described for cgal mutants of Cochliobolus heterostrophus and cpgl mutants of $C$. parasitica. On PSA with up to $1 \%$ sucrose, $\Delta$ bcg 1 mutants grow more slowly and build smaller, more compact colonies than the wild type. The colonies have an atypically even colony margin. Interestingly, $2 \mathrm{mM}$ cAMP fully restored the wild-type colony morphology, whereas higher concentrations of cAMP $(10 \mathrm{mM})$ had rather toxic effects on both the wild-type and the mutant strains (data not shown). In higher organisms, members of the $\mathrm{G} \alpha_{\mathrm{i}}$ family lower intracellular cAMP levels by inhibition (i) of adenylate cyclase activity. Therefore, we expected that the replacement of $b c g l$ would result in an increase of the intracellular cAMP level by removing the inhibitor of adenylate cyclase. However, the opposite occurred. Application of external cAMP overcame the mutant phenotype. A similar stimulatory effect of $\mathrm{G \alpha}_{\mathrm{i}}$ proteins on adenylate cyclase was found for $\mathrm{magB}$ in $M$. grisea; cAMP in the wild type induced and deletion of $m a g B$ blocked appressorium formation (Liu and Dean 1997). In $N$. crassa, $\Delta$ gna- 1 mutants had only 10 to $15 \%$ of the adenylate cyclase activity of the wild type. The steady-state cAMP levels were reduced in the mutants under conditions that result in morphological abnormalities (a solid medium), suggesting a stimulatory role of gna- 1 on the $N$. crassa adenylate cyclase (Ivey et al. 1999). Furthermore, the specific phosphodiesterase also seems to be under the control of gna-1 in $N$. crassa, regulating the intracellular cAMP level in response to extracellular signals.

Apart from regulation of growth rate and colony morphology, BCG-1 was also shown to have a major role in the colonization of host tissue in tomato and beans.

The $B$. cinerea bmpl gene encoding a MAP kinase was recently shown to be essential for plant infection. Conidia of $\Delta$ bmp1 mutants germinated but failed to penetrate and macerate plant tissue and appeared to be unable to infect through wounds (Zheng et al. 2000). In contrast to bmpl mutants, bcgl knock-out mutants sporulate, germinate, and are able to penetrate intact tomato and bean leaves. However, after forming primary lesions, the fungus completely stops invasion of plant tissue; secondary lesions were not observed even 2 weeks postinoculation. In addition, $\Delta \mathrm{bcg} 1$ mutants failed to secrete proteases into the medium, suggesting that the BCG1 protein may also control expression and secretion of hydrolytic enzymes that are involved in plant penetration. A similar result was described for $C$. parasitica. In addition to marked reduction in growth and loss of pathogenicity, deletion of cpgl also resulted in drastic reduction of extracellular laccase activity (Gao and Nuss 1996).

Interestingly, cAMP did not restore the ability to secrete extracellular proteases, demonstrating that BCG1 is involved at least in one additional signaling cascade beside the cAMP pathway. 
On the other hand, deletion of $b c g 2$ had no obvious effect on growth, conidiation, or protease secretion, similar to MAGC, which belongs to the same group of fungal $\mathrm{G} \alpha$ proteins. However, BCG2 is necessary for a normal colonization rate; loss of BCG2 leads to significant retardation of fungal development in planta.

Our results indicate that BCG1 and BCG2 control different signal transduction pathways in $B$. cinerea. Because neither mutant is completely apathogenic and unable to penetrate (such as the bmpl mutant, discussed above), it is possible to compare in planta gene expression of wild-type and mutant strains. This could uncover the target genes of the two G $\alpha$ proteins, allowing the identification of whole sets of genes involved in plant-pathogen interaction in B. cinerea.

\section{MATERIALS AND METHODS}

\section{Strains and growth conditions.}

$B$. cinerea strain B05.10 was derived from the wild-type strain SAS 56 treated with benomyl for haploidization (Quidde et al. 1999). This strain and resulting transformants were grown on PSA containing $0.5,1.0,1.5$, or $2 \%$ sucrose at $20^{\circ} \mathrm{C}$ under irradiation for conidiation.

For DNA isolation, Gamborg's B5 medium (Duchefa Biochemie BV, Haarlem, The Netherlands), supplemented with $0.3 \%$ glucose, was inoculated with $10^{7}$ conidia per $100 \mathrm{ml}$. After $48 \mathrm{~h}$ of growth on a rotary shaker at $180 \mathrm{rpm}$ at $20^{\circ} \mathrm{C}$, mycelium was harvested, washed with sterile water, and lyophilized. For DNA minipreps, mycelium was grown for 3 to 4 days at $20^{\circ} \mathrm{C}$ on complex medium agar (Pontecorvo et al. 1953) with a cellophane overlay. Single-spore isolates were obtained by spreading $100 \mu \mathrm{l}$ of a $10^{2}$-spores $/ \mathrm{ml}$ spore solution on a PSA plate containing $70 \mu \mathrm{g}$ of hygromycin B per ml (Calbiochem-Novabiochem, Bad Soden, Germany) or $40 \mu \mathrm{g}$ of phleomycin per ml (Cayla, Toulouse, France). The conidia were germinated for $24 \mathrm{~h}$ and single colonies transferred individually to new plates with the corresponding antibiotic.

\section{Cloning of the $b c g 1$ and $b c g 2$ genes.}

bcgl was cloned by screening a genomic $\lambda$-EMBL3 library of $B$. cinerea strain SAS 56 (Quidde et al. 1999) with the $C$. parasitica cpgl gene (Choi et al. 1995) as a heterologous probe. Positive phages were purified in a second screening round. Hybridizations were performed at $62^{\circ} \mathrm{C}$. A $b c g 2$ fragment was amplified from B05.10 genomic DNA by PCR with primers Ga-1 and Ga-2. PCR reactions contained $25 \mathrm{ng}$ of DNA, $10 \mathrm{ng}$ of each primer, $0.2 \mathrm{mM}$ dNTPs, and $2 \mathrm{U}$ of Taq polymerase (Red Taq; Sigma-Aldrich, Deisenhofen, Germany) in $50 \mu \mathrm{l}$. PCR was carried out at $94^{\circ} \mathrm{C}$ for $4 \mathrm{~min}$, followed by 30 cycles of $1 \mathrm{~min}$ at $94^{\circ} \mathrm{C}, 1 \mathrm{~min}$ at $55^{\circ} \mathrm{C}$, and $1 \mathrm{~min}$ at $72^{\circ} \mathrm{C}$. The 180-bp PCR fragment was used as a probe for screening the EMBL3 library at $65^{\circ} \mathrm{C}$.

PCR primers are Ga-1, 5'-AAG TGG ATY CAC TGY TTY 6-3', and Ga-2, 5'-RTC RAT CTT GTT SAG GAA-3'.

\section{DNA isolation and Southern blot analysis.}

Fungal genomic DNA was isolated as described by Cenis (1993). Lambda DNA was isolated according to the standard method (Sambrook et al. 1989). Plasmid DNA was isolated using a plasmid DNA preparation kit (Genomed, Bad Oeynhausen, Germany).
Hybridization was carried out in $6 \times \mathrm{SSC}(1 \times \mathrm{SSC}$ is $0.15 \mathrm{M}$ $\mathrm{NaCl}$ plus $0.015 \mathrm{M}$ sodium citrate), $5 \times$ Denhardt's solution, $0.1 \%$ sodium dodecyl sulfate (SDS), and $50 \mathrm{mM}$ phosphate buffer, $\mathrm{pH} 6.6$, at 62 or $65^{\circ} \mathrm{C}$. Membranes were washed twice (with $2 \times$ and $0.1 \times \mathrm{SSC}, 0.1 \% \mathrm{SDS}$ ) before being exposed to autoradiographic film.

\section{RNA isolation and RT-PCR.}

Total RNA was isolated from necrotic spots of Botrytisinfected bean leaves 12, 24, 36, and 48 hpi using the RNA easy extraction kit (Promega, Mannheim, Germany). Five micrograms of total RNA was taken for RT-PCR using a onestep RT-PCR kit (Boehringer, Mannheim, Germany) according to the manufacturer's instructions. The following primers were used for amplification of the fragments of $b c g 1, b c g 2$, and the $B$. cinerea actin (control) genes from cDNA and genomic DNA (Fig. 1): 3, 5'-CTG AAG AGG GAC AAG AFG CTG C-3'; 4, 5'-GAG CGA AGG ACA TCT TGA TCG-3'; $2^{\prime}, 5^{\prime}$-GAG ATG AGA AGG TTA TGC AGC G-3'; 4', 5'-CTT CTG GAT ACC TGA GTC TGC C-3'; actin-F, 5'-TTC CAT TGT CGG TCG TCC CCG-3'; and actin-R, 5'-ACC TGG GTA CAT AGT GGT TCC ACC-3'.

The amplification started with $4 \mathrm{~min}$ at $94^{\circ} \mathrm{C}$, followed by 35 cycles of $1 \mathrm{~min}$ at $94^{\circ} \mathrm{C}, 1 \mathrm{~min}$ at $58^{\circ} \mathrm{C}, 1 \mathrm{~min}$ at $72^{\circ} \mathrm{C}$, and a final $10 \mathrm{~min}$ at $72^{\circ} \mathrm{C}$ before cooling to $18^{\circ} \mathrm{C}$. PCR products were analyzed on a $2 \%$ agarose gel and cloned into the pCR2.1 cloning vector (Invitrogen, Groningen, The Netherlands) for sequencing.

\section{Infection assays on plants and tomato fruits.}

Conidia were collected from 7- to 10-day-old PSA plates and resuspended in Gamborg's B5 medium (Duchefa Biochemie BV) supplemented with $10 \mathrm{mM}$ glucose and $10 \mathrm{mM} \mathrm{KH}_{2} \mathrm{PO}_{4}$, $\mathrm{pH} 6.4$, to a final concentration of $10^{5}$ conidia per ml. Droplets of $5 \mu$ of the conidial suspension of the wild type and transformants were placed on the left and right side of primary leaves of bean plants, respectively. The infected plants and fruits were incubated in a Plexiglas box at $20^{\circ} \mathrm{C}$ with natural illumination conditions. Disease symptoms were scored 2 to 7 days after inoculation.

\section{Construction}

of replacement and complementation vectors.

For construction of the gene replacement vectors $\mathrm{p} \Delta \mathrm{bcg} 1$ and $\mathrm{p} \Delta \mathrm{bcg} 2$, the plasmid pLOF1A (kindly provided by J. van Kan, University of Wageningen, The Netherlands), carrying the $E$. coli hygromycin resistance gene under the control of the Aspergillus nidulans oliC promoter and the $\operatorname{trp} C$ terminator, was used as a basis vector. A 1.8-kb KpnI/SalI fragment from the plasmid pbcg1-Bam ( $5^{\prime}$ region of $\left.b c g 1\right)$ and a $3.0-\mathrm{kb}$ $X b a I$ fragment from the plasmid pbcg1-Xba ( $3^{\prime}$ region of bcg1) were cloned into the corresponding sites of pLOF1A, resulting in the gene replacement vector $\mathrm{p} \Delta \mathrm{bcg} 1$.

For construction of $\mathrm{p} \Delta \mathrm{bcg} 2$, a 1.1-kb PCR fragment was amplified from the $3^{\prime}$ region of the gene $b c g 2$ using the following primers: 5', 5'-GGA GTC GAC TGC TAA CTC TCA TTG-3', and 6', 5'-GGT ACC CGG GGA TCC ACT AG-3'.

Primer $6^{\prime}$ contains an artificial SalI site for further cloning. A 1.6-kb PCR fragment of the $5^{\prime}$ end of bcg2 was amplified using the following primers: $1^{\prime}$, M13 reverse primer, and $3^{\prime}$, 5'-CCA GAT TCT CCA GCA CCT GCA GAC C-3'. 
Both PCR products were cloned into pPCR2.1 (Invitrogen); cut out with KpnI/SalI and XbaI/HindIII, respectively; and cloned into the corresponding sites of pLOF1A. For the construction of the complementation vector pbcg1+, the 2.4-kb SalI fragment from pbcg1 was ligated into the SalI-digested pbcg1-Bam.

\section{Transformation.}

Protocols for protoplast formation and transformation were adapted from established procedure (ten Have et al. 1998). Mycelia were prepared as described above and harvested using sterile filters. After washing with $\mathrm{KCl}$ buffer $(0.6 \mathrm{M} \mathrm{KCl}$, $50 \mathrm{mM} \mathrm{CaCl}$ ), $2 \mathrm{~g}$ of mycelium was suspended in $20 \mathrm{ml}$ of $\mathrm{KCl}$ buffer with 0.5\% Novozyme 234 (Interspex Products Inc.). After $1 \mathrm{~h}$ of agitation at $28^{\circ} \mathrm{C}, 120 \mathrm{rpm}$, protoplasts were collected using sterile cheesecloth and pelleted by centrifugation $(1,500 \times g)$ for $8 \mathrm{~min}$. Protoplasts were resuspended in $\mathrm{KCl}$ buffer and adjusted to a concentration of $1 \times 10^{8}$ protoplasts per ml. For knock-out experiments, 10 to $15 \mu \mathrm{g}$ of the $K p n I$-linearized vector $\mathrm{p} \Delta \mathrm{bcg} 1$ or of a HindIII/SmaI fragment, excised from $\mathrm{p} \Delta \mathrm{bcg} 2$, was added to $10^{7}$ protoplasts in $50 \mu \mathrm{l}$ of $0.6 \mathrm{M} \mathrm{KCl}, 50 \mathrm{mM} \mathrm{CaCl}_{2}$.

For complementation of bcgl knock-out transformants, a cotransformation of the mutant strain $\Delta$ bcg1-1 with the plasmid pbcg- $1+$ containing the whole $b c g l$ gene with $2.5 \mathrm{~kb}$ of the $5^{\prime}$ noncoding region and plasmid pLOF2 (kindly provided by J. van Kan, University Wageningen), carrying the phleomycin resistance cassette with the A. nidulans oliC promoter and the $\operatorname{trp} C$ terminator, was performed.

Homologous integration events were identified by PCR, using the following primers: for bcg1, 1, 5' CGC TAT AAC CAA ATC ATC CTT CTT CCC ATG C 3', and 6, 5' GGT ACT GCC CCA CTT AGT GGC AGC TCG CG 3'; and for bcg2, 7', 5' GGG CTG TCG GCA CAG GTT TCT GG 3', and 6, 5' GGT ACT GCC CCA CTT AGT GGC AGC TCG CG 3'.

Transformants were screened for integration of the entire bcgl fragment by PCR using the following primers: 2, 5' CCT TCA GCT CAC CAA ACTTCC C3'; 4, 5' CCT TCA GCT CAC CAA ACTTCC C3'; 3, 5' CCT TCA GCT CAC CAA ACTTCC C3'; and 5, 5' CCC CAA CGA GCC ATC CAA CAA TCA CC $3^{\prime}$.

\section{Protease assay.}

The extracellular protease assay was performed by incubating $B$. cinerea strain $\mathrm{B} 05.10$ and $\Delta \mathrm{bcg} 1$ and $\Delta \mathrm{bcg} 2$ transformants on casein agar (12.5 ml per liter of milk, $2 \mathrm{~g}$ per liter of $\mathrm{K}_{2} \mathrm{HPO}_{4}$, and $16 \mathrm{~g}$ of agar, $\mathrm{pH} 6.5$ ) for 2 to 3 days at $20^{\circ} \mathrm{C}$. Secretion of proteases resulted in the formation of transparent halos around the colonies.

\section{SEM.}

Bean leaves were inoculated with conidia of the wild-type and the mutant strains as described above. After $84 \mathrm{~h}$, the lesions were cut out and directly infiltrated with fixing solution (1\% glutaraldehyde, $5 \%$ formaldehyde in $25 \mathrm{mM}$ sodium phosphate buffer, $\mathrm{pH}$ 7.0) for 10 to $30 \mathrm{~min}$. After infiltration, the leave disks were incubated in fixing solution for $1 \mathrm{~h}$ at room temperature and then for $30 \mathrm{~min}$ at $4^{\circ} \mathrm{C}$. The samples were washed three times with $25 \mathrm{mM}$ sodium phosphate buffer ( $\mathrm{pH} \mathrm{7.0)}$ at $4{ }^{\circ} \mathrm{C}$ for $30 \mathrm{~min}$, followed by 30-min dehydration periods in graded ethanols $(30,50,70,96$, and 99\%).
The dehydrated samples were dried at critical point. The material was then stuck onto aluminum plates $(8 \times 12 \mathrm{~mm})$ and covered with gold under a cryon atmosphere (Leitz, Wetzlar, Germany). A Hitachi S-530 REM (Hitachi, Tokyo) was used for microscopy. Photographs were made on Agfa Pan 100 films (Agfa, Köln, Germany).

\section{ACKNOWLEDGMENTS}

We thank D. Nuss for providing us with the Cryphonectria parasitica cpgl gene, J. van Kan for vectors pLOF1A and pLOF2, S. Moore for critical reading, and $\mathrm{B}$. Berns for typing the manuscript.

\section{LITERATURE CITED}

Aimi, T., Kano, S., Wang, Q., and Morinaga, T. 2001. Molecular cloning of three genes encoding $\mathrm{G}$ protein alpha subunits in the white root rot fungus, Rosellinia neactrix. Biosci. Biotechnol. Biochem. 65:678-682.

Bölker, M. 1998. Sex and crime: Heterotrimeric G proteins in fungal mating and pathogenesis. Fungal Genet. Biol. 25:143-156.

Cenis, J. L. 1993. Rapid extraction of fungal DNA for PCR amplification. Nucleic Acids Res. 20:2380.

Choi, G. H., Chen, B., and Nuss, D. L. 1995. Virus-mediated or transgenic suppression of a G-protein alpha subunit and attenuation of fungal virulence. Proc. Natl. Acad. Sci. U.S.A. 92:305-309.

Gao, S., and Nuss, D. L. 1996. Distinct roles for two G protein alpha subunits in fungal virulence, morphology and reproduction revealed by targeted gene disruption. Proc. Natl. Acad. Sci. U.S.A. 93:1412214127.

Horwitz, B. A., Sharon, A., Lu, S.-W., Ritter, V., Sandrock, T. M., Yoder, O. C., and Turgeon, B. G. 1999. A G protein alpha subunit from Cochliobolus heterostrophus involved in mating and appressorium formation. Fungal Genet. Biol. 26:19-32.

Ivey, F. D., Yang, Q., and Borkovich, K. A. 1999. Positive regulation of adenyl cyclase activity by a $\mathrm{G}_{\mathrm{i}}$ homolog in Neurospora crassa. Fungal Genet. Biol. 26:48-61.

Kays, A. M., Rowley, P. S., Baasiri, R. A., and Borkovich, K. A. 2000. Regulation of conidiation and adenylyl cyclase levels b the G $\alpha$ protein GNA-3 in Neurospora crassa. Mol. Cell. Biol. 20:7693-7705.

Lichter, A., and Mills, D. 1997. Fil1, a G-protein $\alpha$-subunit that acts upstream of cAMP and is essential for dimorphic switching in haploid cell of Ustilago hordei. Mol. Gen. Genet. 256:426-435.

Liu, S., and Dean, R. A. 1997. G protein $\alpha$ subunit genes control growth, development, and pathogenicity of Magnaporthe grisea. Mol. PlantMicrobe Interact. 10:1075-1086.

Liu, S., Oeljeklaus, S., Gerhardt, B., and Tudzynski, B. 1998. Purification and characterization of glucose oxidase of Botrytis cinerea. Physiol. Mol. Plant Pathol. 53:123-132.

Pennington, S. R. 1994. GTP-binding proteins I: Heterotrimeric G proteins. Protein Profiles 1:169-233.

Pontecorvo, G. V., Poper, J. A., Hemmonns, L. M., Mac Donald, K. D., and Buften, A. W. J. 1953. The genetics of Aspergillus nidulans. Adv. Genet. 141:141-238.

Quidde, T., Büttner, P., and Tudzynski, P. 1999. Evidence for three different specific saponin-detoxifying activities in Botrytis cinerea and cloning of a gene coding for a putative avenacinase. Eur. J. Plant Pathol. 105:273-283.

Rebordinos, L., Cantoral, J. M., Prieto, M. V., Hanson, J. R., and Collado, I. G. 1996. The phytotoxic activity of some metabolites of Botrytis cinerea. Phytochemistry 42:383-387.

Regenfelder, E., Spellig, T., Hartmann, A., Lauenstein, S., Bölker, M., and Kahmann, R. 1997. G proteins in Ustilago maydis: Transmission of multiple signals? EMBO (Eur. Mol. Biol. Organ.) J. 16:1934-1942.

Sambrook, J., Fritsch, E. F., and Maniatis, T. 1989. Molecular Cloning: A Laboratory Manual, 2nd ed. Cold Spring Harbor Laboratory, Cold Spring Harbor, NY, U.S.A.

Schoonbeek, H., Del Sorbo, G., and De Waard, M. A. 2001. The ABC transporter BcatrB affects the sensitivity of Botrytis cinerea to the phytoalexin resveratrol and the fungicide fenpiclonil. Mol. PlantMicrobe Interact. 14:562-571.

Strathmann, M., Wilkie, T. M., and Simon, M. I. 1989. Diversity of the 
G-protein family: Sequences from five additional $\alpha$ subunits in the mouse. Proc. Natl. Acad. Sci. U.S.A. 86:7407-7409.

ten Have, A., Mulder, W., Visser, J., and van Kan, J. A. L. 1998. The endopolygalacturonase gene $B c p g 1$ is required for full virulence of Botrytis cinerea. Mol. Plant-Microbe Interact. 11:1009-1016.

Truesdell, G. M., Yang, Z., and Dickman, M. B. 2000. A G $\alpha$ subunit gene from the phytopathogenic fungus Colletotrichum trifolii is required for conidial germination. Physiol. Mol. Plant Pathol. 56:131140.

Turner, G. E., and Borkovich, K. A. 1993. Identification of a G protein $\alpha$ subunit from Neurospora crassa that is a member of the Gi family. $\mathrm{J}$.
Biol. Chem. 268:14805-14811.

von Tiedemann, A. 1997. Evidence for a primary role of active oxygen species in induction of host cell death during infection of bean leaves with Botrytis cinerea. Physiol. Mol. Plant Pathol. 50:151-166.

Yu, J.-H., Wieser, J., and Adams, T. H. 1996. The Aspergillus FLBA RGS domain protein antagonizes G-protein signaling to block proliferation and allow development. EMBO (Eur. Mol. Biol. Organ.) J. 15: 5184-5190.

Zheng, L., Campbell, M., Murphy, J., Lam, S., and Xu, J.-R. 2000. The $B M P 1$ gene is essential for pathogenicity in the gray mold fungus $B o-$ trytis cinerea. Mol. Plant-Microbe Interact. 13:724-732. 